\title{
Efeito da L-Glutamina sobre o perfil leucocitário e a função fagocítica de macrófagos de ratos estressados
}

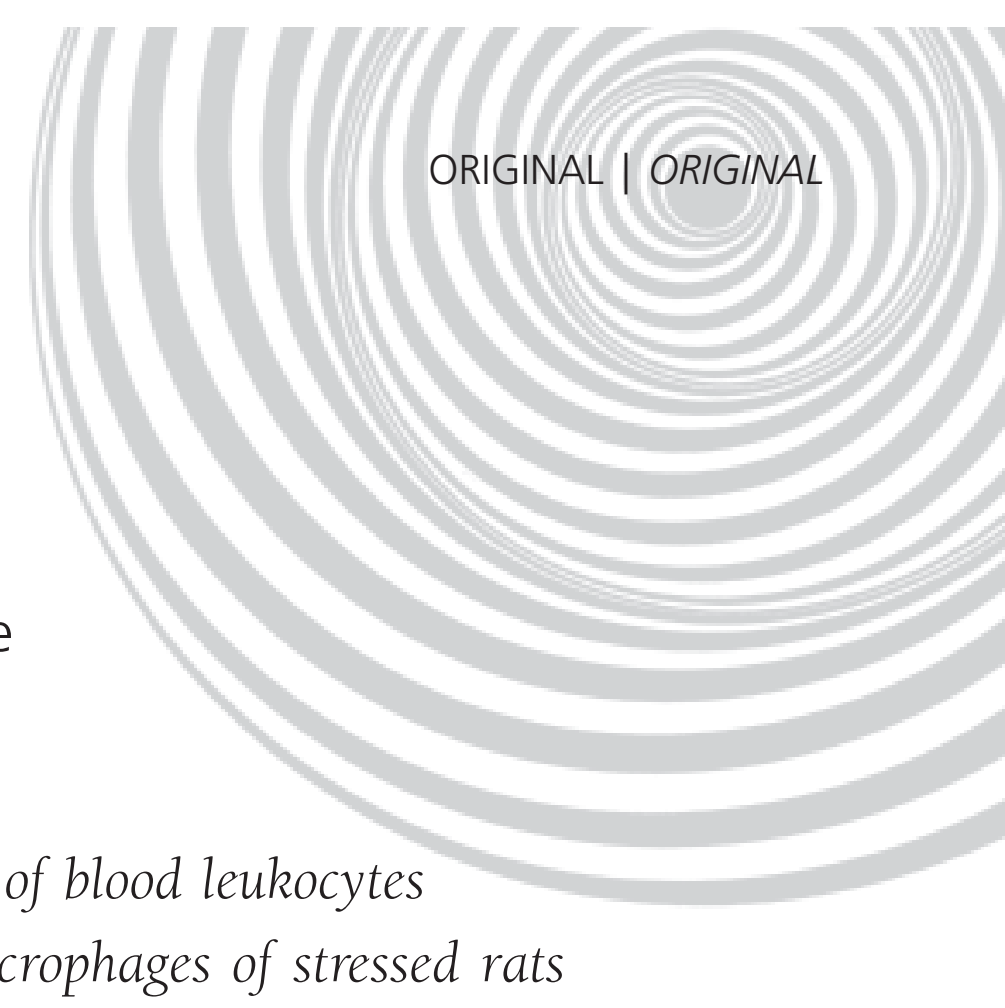

\author{
Carol Góis LEANDRO' \\ Elizabeth do NASCIMENTO² \\ Maria Magdala AZEVEDO \\ Andrezza VIEGAS² \\ Camila ALBUQUERQUE ${ }^{2}$ \\ Cláudio Barnabé CAVALCANTI \\ Raul MANHÃES-DE-CASTRO \\ Célia Maria Machado Barbosa de CASTRO 3
}

RE S U M O

\section{Objetivo}

Verificar o efeito da administração intraperitoneal de glutamina, prévia a uma situação de estresse, sobre o número de leucócitos do sangue e sobre a taxa de fagocitose de macrófagos alveolares de ratos.

\section{Métodos}

Ratos machos Wistar aos 90 dias de idade foram divididos em controle $(C, n=15)$, estresse $(C+E, n=16)$, glutamina ( $G L N, n=15)$ e glutamina e estresse $(G L N+E, n=15)$ e mantidos em condições padronizadas. Os grupos GLN e GLN+E receberam, via intraperitoneal ( $\mathrm{i}$ ip), L-glutamina $(0,2 \mathrm{~g} / \mathrm{kg}$ de peso corporal) durante 10 dias. Seus pares receberam, v ip, L-glicina $(0,4 \mathrm{~g} / \mathrm{kg}$ do peso corporal). Os animais $\mathrm{C}+\mathrm{E}$ e $\mathrm{GLN}+\mathrm{E}$ foram submetidos ao estresse agudo de contenção (40min, após $24 \mathrm{~h}$ da última intervenção com o aminoácido). Leucócitos totais foram contados em hemocitômetro e os diferenciais por esfregaço sanguíneo. Para avaliação da taxa de fagocitose, os macrófagos alveolares ( $1 \times 10^{6}$ em meio de cultura RPMI) e fungos S. cerevisiae $\left(1 \times 10^{7}\right)$ foram incubados em estufa $\left(37^{\circ} \mathrm{C}, 5 \% \mathrm{CO}_{2}\right)$ durante 1 hora. Foram utilizados os testes estatísticos análise de variância (ANOVA) e o teste de Tukey, valores expressos em média do percentual e desvio-padrão.

\footnotetext{
1 Escola Superior de Educação Física, Universidade de Pernambuco. Rua Arnóbio Marques, 310, 50100-130, Santo Amaro, Recife, PE, Brasil. Correspondência para/Correspondence to: C.G. LEANDRO.E-mail: <cleandro@esef.upe.br>.

2 Laboratório de Fisiologia da Nutrição, Departamento de Nutrição, Universidade Federal de Pernambuco. Recife, PE, Brasil.

${ }^{3}$ Laboratório de Imunopatologia Keizo Asami, Setor de Microbiologia, Universidade Federal de Pernambuco. Recife, PE, Brasil.
} 
438 | C.G. LENADRO et al.

\section{Resultados}

Comparado ao grupo controle, o C+E apresentou diminuição no número de leucócitos e linfócitos e aumento no número de neutrófilos $(p<0,05)$. A taxa de fagocitose de macrófagos diminuiu no grupo $C+E$, quando comparada ao controle $(p<0,05)$. Esta diminuição permaneceu no grupo $G L N+E$ e não foi alterada no grupo $\operatorname{GLN}(p>0,05)$.

\section{Conclusão}

A administração, via ip, de glutamina não atenua o efeito do estresse no número de leucócitos e na função fagocítica de macrófagos alveolares em ratos.

Termos de indexação: estresse; glicina; glutamina; imunonutrientes.

\section{A B S T R A C T}

\section{Objective}

To verify the effect of the intraperitoneal administration of glutamine previously to a stress situation on the number of blood leukocytes and on the alveolar macrophage phagocytosis rate in rats.

\section{Methods}

Male Wistar rats (90 days old) were divided into four experimental group; control $(C, n=15)$, stress $(C+E, n=16)$, glutamine (GLN, $n=15)$, and glutamine + stress $(G L N+E, n=16)$ experimental groups and kept under standard conditions. The GLN and GLN+E groups received L-glutamine $(0,2 \mathrm{~g} / \mathrm{kg}$ of body weight) intraperitoneally (ip) during 10 days. Their controls received L-glicine $(0,4 \mathrm{~g}$ of aal $\mathrm{kg}$ of body weight) ip. The animals of $C+E$ and GLN+E groups were submitted to acute restraint stress (40min, 24h after the last amino acid intervention). Total leukocytes were counted by hemocytometer and for differential leukocytes, count smears were used. To evaluate the phagocytosis rate, the alveolar macrophages $\left(1 \times 10^{6}\right.$ in a RPMI culture) and S. cerevisiae $\left(1 \times 10^{7}\right)$ yeast were incubated in an incubator $\left(37^{\circ} \mathrm{C}, 5 \% \mathrm{CO}_{2}\right)$ for one hour. The ANOVA and TUKEY (post-hoc) tests were used for the statistical analysis of variance and the values were expressed in mean and standard deviation.

\section{Results}

When compared with the control group, the C+E group presented a decrease in the number of leukocytes and lymphocytes and an increase in the number of neutrophils $(p<0.05)$. The alveolar macrophage phagocytosis rate decreased in the $C+E$ group when compared with the control $(p<0.05)$. This decrease remained in the GLN+E group and did not change in the GLN group ( $p>0.05)$.

\section{Conclusion}

The administration of L-glutamine intraperitoneally does not attenuate the effect of stress on the number of blood leukocytes and phagocytic function of alveolar macrophages in rats.

Indexing terms: glutamine; glycine; immunonutrients; stress.

\section{N T R O D U Ç Ã O}

A relação entre disponibilidade de aminoácidos ou de outros substratos metabólicos, e o bom funcionamento do sistema de defesa do organismo vem sendo estabelecida ${ }^{1,2}$. Consideráveis esforços são realizados na identificação de substâncias capazes de melhorar a resposta imune, com os chamados imunonutrientes ${ }^{3,4}$. A imunonutrição constitui um novo campo de investigação em nutrição ${ }^{4}$. Atualmente, tem-se dado muita atenção à influência dos nutrientes na fase inflamatória aguda, em particular, do trato intestinal ${ }^{5}$.

Estados hipercatabólicos do organismo são observados quando não há adaptação a agentes estressores como, por exemplo: o exercício exaustivo ou prolongado, as pós-cirurgias, o transplante de medula óssea e em outras situações de comprometimento da homeostase orgânica ${ }^{4,6,7}$. O estresse agudo de contenção tem sido utilizado como modelo indutor de estresse psicológico e 
desencadeia alterações imunes e um padrão de secreção hormonal similares aos demais tipos de estímulos indutores de estresse ${ }^{8}$.

A modulação do sistema imunológico, induzida pelo estresse já é um fenômeno bem conhecido ${ }^{8,9}$, estando descrita a ocorrência de neutrofilia, linfopenia e monocitose a seguir à exposição ao estímulo indutor da situação de estresse $^{6}$. As funções anti-microbiana e antitumoral de macrófagos/monócitos parecem, também, diminuir ${ }^{10}$. A diminuição dessas funções tem sido associada a uma maior incidência de infecções e ao alastramento de tumores em animais submetidos a condições de estresse prolongado ${ }^{10,11}$.

No hipercatabolismo a glutamina é reconhecida como aminoácido "condicionalmente essencial", e tem despertado interesse dos pesquisadores por sua capacidade de interferir no funcionamento de células do sistema imune ${ }^{8,9,11}$. Por exemplo, os macrófagos utilizam glutamina para realização da função fagocítica e citotóxica ${ }^{12}$. Esse aminoácido também é utilizado em altas taxas para proliferação de linfócitos e atividade fagocítica de neutrófilos ${ }^{2,13}$.

O aumento dos níveis plasmáticos de corticosterona, associado ao estresse agudo, pode exacerbar a gliconeogênese, causando degradação protéica acompanhada de padrão específico de liberação de aminoácidos para o sangue $^{12}$. Dos aminoácidos mobilizados do meio intra para o extracelular, em resposta ao estresse, a glutamina parece ser um dos primeiros ${ }^{13,14}$. Ao mesmo tempo, o estresse pode provocar um aumento na captação da glutamina pelos rins, fígado e intestino ${ }^{7}$. Se há um aumento na utilização e diminuição na demanda, a suplementação poderia ser um fator importante ${ }^{15}$. Estudos recentes demonstram que a suplementação oral ou intravenosa de glutamina tem ação imunoestimulatória em linfócitos e neutrófilos ${ }^{14}$. Apesar das evidências da importância da suplementação da glutamina, Newsholme ${ }^{7}$ recomenda maiores investigações sobre a influência deste aminoácido sobre o sistema imune in vivo.
Este estudo teve por objetivo verificar o efeito da administração via intraperitoneal de glutamina, prévia a uma situação de contenção aguda indutora de estresse, sobre o perfil leucocitário do sangue periférico e a taxa de fagocitose de macrófagos alveolares de ratos.

\section{MÉ T O D O S}

Ratos albinos machos da linhagem Wistar aos 90 dias de idade, com um peso corporal de 309 e desvio-padrão (dp) de 8,3g foram divididos em dois grupos: controle- $C(n=31)$ e administrados com L-glutamina - $\mathrm{GLN}(\mathrm{n}=30)$. Os animais foram mantidos na Colônia do Departamento de Nutrição, Universidade Federal de Pernambuco em gaiolas coletivas com temperatura ambiente próxima de $22^{\circ} \mathrm{C}$ e receberam dieta balanceada (52\% de carboidratos, $21 \%$ de proteínas e $4 \%$ de lipídeos - Nuvilab CR1-Nuvital ${ }^{\circledR}$ ) e água ad libitum. Manteve-se um ciclo claro/escuro de $12 / 12$ horas (claro das $6 \mathrm{~h}$ às $18 \mathrm{~h}$; escuro das $18 \mathrm{~h}$ às $6 \mathrm{~h}$ ). Para o grupo glutamina foi utilizada a dosagem de $0,2 \mathrm{~g} / \mathrm{kg}$ de peso do animal, via intraperitoneal, durante 10 dias, segundo a dose utilizada no estudo de Kew e colaboradores ${ }^{10}$. Para os animais $C$, foi administrado, via intraperitoneal, o aminoácido L-glicina na dose de $0,4 \mathrm{~g} / \mathrm{kg}$ do peso do animal, de forma a manter o balanço nitrogenado ${ }^{12}$. O peso corporal dos animais foi monitorado diariamente durante o período do experimento. Este estudo foi apro-vado pela Comissão Ética em Experimentação Animal do Centro de Ciências Biológicas da Universidade Federal de Pernambuco, e seguiu as normas sugeridas pelo Comitê Brasileiro de Experimentação Animal (COBEA).

\section{Indução de estresse}

Logo após os 10 dias de administração do aminoácido, os dois grupos foram subdivididos, 
dando origem a quatro grupos experimentais, de acordo com a posterior sujeição, ou não, à situação de contenção (indutora de estresse). Assim, os animais controle foram divididos em 2 grupos: $C(n=15)$, sujeito à administração da L-glicina, e o grupo $C+E(n=16)$, sujeito à administração da L-glicina e à situação de contenção aguda. Da mesma forma, os animais que receberam glutamina também foram divididos em 2 grupos: $G L N(n=15)$, sujeito à administração de L-glutamina, e o grupo $G L N+E(n=15)$, sujeito à adminstração de L-glutamina seguida por exposição à contenção aguda. Para indução da situação de contenção aguda, nos grupos $\mathrm{C}+\mathrm{E} e$ $\mathrm{GLN}+\mathrm{E}$, os animais foram colocados num tubo cilíndrico $(24 \mathrm{~cm}$ de comprimento por $8 \mathrm{~cm}$ de largura), onde permaneceram contidos durante 40 minutos, de acordo com a técnica proposta por Selye ${ }^{16}$. A contenção dos animais teve lugar 24 horas após o último dia de administração do aminoácido. Uma pequena alíquota de sangue $(0,2 \mathrm{ml})$ foi retirada imediatamente antes e após o período de contenção. A seguir, os animais foram anestesiados e as células (macrófagos alveolares) retiradas para análise. O restante dos animais foi sujeito aos mesmos procedimentos (coleta de sangue, anestesia e coleta de células), 25 horas após o último dia de administração do aminoácido (grupos C e GLN).

\section{Parâmetros analisados}

- Leucograma: as amostras de sangue foram diluídas (1:200) em solução de TURK (ácido acético a $3 \%)^{17}$. Os leucócitos foram contados em hemocitômetro com auxílio do microscópio óptico. O resultado foi obtido com a fórmula: Leucócitos $\times \mathrm{mm}^{3}$ de sangue $=($ LC. $\times 20 \times 10) / 4$. Na qual: $\mathrm{LC}=$ número total de leucócitos contados em $4 \mathrm{~mm}^{2} ; 4=$ fator de conversão para $1 \mathrm{~mm}^{3} ; 20=$ fator de conversão da diluição utilizada; $10=$ fator de conversão para $1 \mathrm{~mm}^{3}$ (profundidade da lâmina). Para contagem diferencial de leucócitos foi utilizada a técnica do esfregaço sanguíneo.
Para coloração do esfregaço foi utilizado o kit Panótico Rápido LB (Laborclin Ltda, BR). Os diferentes elementos foram contabilizados por contador eletrônico KACIL com teclas correspondentes a cada tipo de célula.

- Macrófagos alveolares: os animais foram anestesiados via ip com solução cloralose a 0,5\% e uretana a $12,5 \%$, na dose de $0,8 \mathrm{ml}$ por $100 \mathrm{~g}$ de peso do animal. $O$ exudato broncoalveolar foi realizado por injeção de soro fisiológico (SF) à temperatura ambiente, através de cânula plástica inserida na traquéia ${ }^{18}$. Várias alíquotas de $2 \mathrm{ml}$ de SF foram injetadas e imediatamente aspiradas. Ao final, coletou-se um volume de $20 \mathrm{ml}$ de lavado broncoalveolar por animal, que corresponderia a cerca de $3 \times 10^{6}$ macrófagos em cada lavado.

- Taxa de fagocitose: foram utilizados fungos (Saccharomyces sp.) para avaliar a taxa de fagocitose de acordo com a técnica de Malageño et al. ${ }^{19}$. Os fungos foram lavados 2 vezes com solução tampão fosfato (PBS), contados $10^{7}$ células e, em seguida, misturados na suspensão de macrófagos $(1 \times 10 \% / \mathrm{ml} \mathrm{em} \mathrm{meio} \mathrm{de} \mathrm{cultura}$ completo, RPMI 1640) recuperados do lavado broncoalveolar. As células (macrófagos e fungos) foram distribuídas em lâminas de microscopia óptica e incubados a $37^{\circ} \mathrm{C}$, em atmosfera úmida durante 1 hora. As lâminas foram lavadas com água destilada e utilizado o kit panótico rápido para a coloração. As lâminas foram lidas ao microscópio óptico com objetiva de 100x sob imersão. A taxa de fagocitose foi obtida como percentual de macrófagos, que englobaram o fungo em uma contagem total de 100 células.

A estatística descritiva está expressa em média e desvio-padrão. Para comparar os valores pré e pós-procedimento da situação indutora de estresse foi utilizado o teste " $t$ " test student pareado. Para comparação entre os grupos foi utilizada a análise de variância (ANOVA). Quando a ANOVA revelou diferença significativa, utilizou-se o teste de Tukey de comparação múltipla. A significância foi mantida em $5 \%$ em todos os casos. 


\section{RESULTADOS}

Os animais que receberam glutamina apresentaram um maior ganho de peso corporal a partir do sexto dia de administração do aminoácido, comparativamente ao grupo controle (Figura 1).

Na contagem total de leucócitos do sangue periférico, o grupo $C+E$ apresentou uma diminuição comparativamente aos valores pré estresse de contenção (pré= $=12,6 \mathrm{dp}=0,7$ e pós $=7,3 \mathrm{dp}=0,4$ ) (Figura 2). Da mesma forma, essa diminuição foi verificada quando todos os grupos estudados foram comparados no segundo momento da coleta de sangue $(C=11,9 d p=1,2 ; C+E=7,3 d p=1,4$; $\mathrm{GLN}=12,0 \mathrm{dp}=1,3 ; \mathrm{GLN}+\mathrm{E}=7,6 \mathrm{dp}=1,2)$. Vale salientar que os animais GLN+E apresentaram diminuição na contagem total de leucócitos quando os valores pré e pós-estresse de contenção foram comparados (pré $=11,9 \mathrm{dp}=1,2$ e pós $=7,6$ $\mathrm{dp}=1$,2) (Figura 2).

Relativamente à contagem diferencial de leucócitos, quando comparado ao grupo $\mathrm{C}$, o grupo $C+E$ apresentou uma diminuição no percentual de linfócitos $(C=76,7 \mathrm{dp}=1,2$ e $\mathrm{C}+\mathrm{E}=68,8 \mathrm{dp}=1,4 p<0,05)$ e um aumento no número de neutrófilos $(C=19,7 \mathrm{dp}=2,1$ e $C+E=27,7 d p=1,3 p<0,05)$. Este perfil leucocitário foi também verificado no grupo $\mathrm{GLN}+\mathrm{E}$ (neutrófilos $=25,4 \mathrm{dp}=2,0$ e linfócitos $=69,8 \mathrm{dp}=2,7$ $p<0,05)$, quando comparado ao $C$ (Figura 3 ).

Nas demais células analisadas, monócitos, eosinófilos e basófilos, não houve diferença entre os grupos.

Os animais $\mathrm{C}+\mathrm{E}$ apresentaram uma diminuição na taxa de fagocitose quando comparado ao $C(21,0 d p=1,3$ e $26,7 d p=1,9$, respectivamente, $p<0,05)$. O grupo glutamina permaneceu com os valores similares ao controle $(25,8 d p=1,9)$. $E$, finalmente, o grupo $G L N+E$ apresentou uma diminuição em relação aos grupos controle e glutamina $(21,8 \mathrm{dp}=2,2)$ (Figura 4).

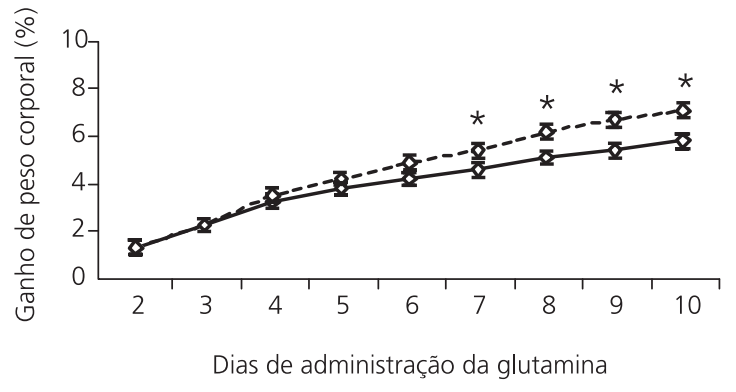

$\leadsto$ Controle $-\diamond--$ Glutamina

Figura 1. Valores médios e desvio-padrão do ganho de peso corporal dos animais dos grupos controle e injetados ip com Glutamina (0,2g/kg de peso corporal). Recife, 2003.

Nota: Teste t-student para comparação entre os grupos. $\left.{ }^{*} p<0,05\right)$

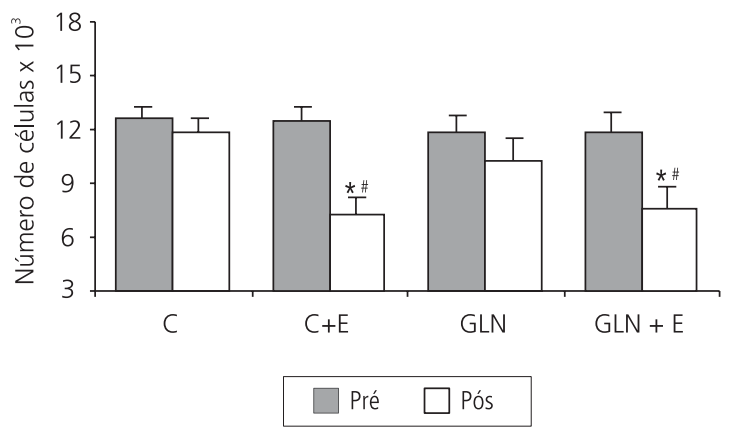

Figura 2. Valores médios e desvio-padrão da contagem total de leucócitos dos animais dos grupos estudados. Recife, 2003.

Nota: $C=$ controle; $C+E=$ estresse; $G L N=$ glutamina e $G L N+E=$ glutamina e estresse. Teste " $t$ " student pareado para comparação dos valores médios pré e pós administração do aminoácido. Teste ANOVA, seguido do teste de Tukey para comparação entre os grupos pós-administração do aminoácido. * $p<0,05$ na comparação dos valores pré e pós. $\# p<0,05$, quando comparado ao grupo $C$ ).

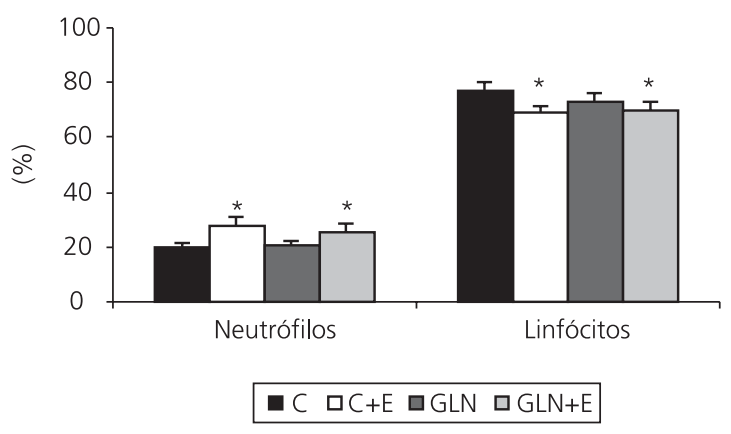

Figura 3. Valores médios e desvio-padrão da contagem total de leucócitos dos animais dos grupos estudados. Recife, 2003.

Nota: $\mathrm{C}=$ controle; $\mathrm{C}+\mathrm{E}=$ estresse; $\mathrm{GLN}=$ glutamina e $\mathrm{GLN}+\mathrm{E}=$ glutamina e estresse. Teste ANOVA, seguido do teste de Tukey. ${ }^{*} p<0,05$, quando comparado ao grupo $C$. 


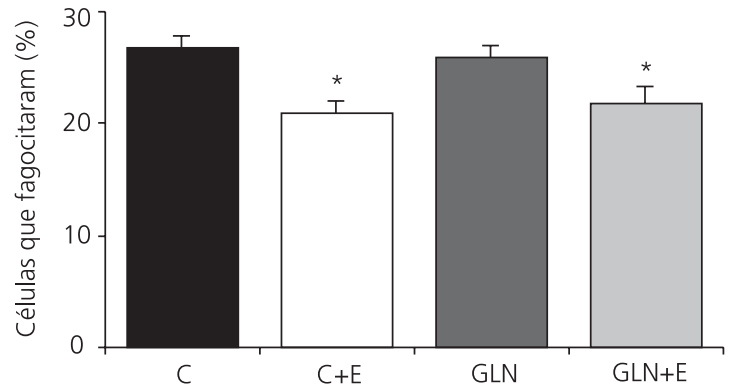

Figura 4. Valores expressos em média e desvio-padrão da percentagem de células que fagocitaram o fungo dos animais dos grupos estudados. Recife, 2003.

Nota: $\mathrm{C}=$ controle; $\mathrm{C}+\mathrm{E}=$ estresse; $\mathrm{GLN}=$ glutamina e $\mathrm{GLN}+\mathrm{E}=$ glutamina e estresse. (Teste ANOVA, seguido do teste de Tukey. ${ }^{*} p<0,05$ quando comparados ao grupo C).

\section{I S C U S S Ã O}

A relação bidirecional entre os sistemas neuro-endócrino e imunológico sugere que alterações fisiológicas em resposta a um estressor tenham repercussões na eficiência do mecanismo de defesa orgânico ${ }^{20}$. Neste estudo, a situação de contenção promoveu alterações nos parâmetros imunes avaliados, muitos dos quais podendo ser indicadores de imunossupressão, como a diminuição do percentual de linfócitos do sangue e da função fagocítica de macrófagos. Na presença desse estímulo estressor, os animais apresentaram um aumento no percentual de neutrófilos do sangue. Estes resultados corroboram estudos previamente realizados com o mesmo modelo de indução de estresse ${ }^{21-23}$. A diminuição observada na dinâmica de células parece estar mais correlacionada com a liberação dos hormônios do estresse, resultando numa redistribuição de células ${ }^{24}$, do que com a disponibilidade de nutrientes ${ }^{22}$. Apesar de não termos avaliado os níveis plasmáticos de glicocorticóides, outros estudos utilizando o mesmo modelo de indução de estresse já o fizeram ${ }^{18}$.

A indução de estresse por contenção também resultou numa diminuição da atividade fagocítica de macrófagos broncoalveolares. Estes resultados corroboram os de Moinard et al. ${ }^{12}$, que verificaram uma diminuição da atividade fagocítica de macrófagos peritoniais de ratos fisicamente estressados em resposta a estímulo com Zimosan. Também Nascimento et al. ${ }^{23}$ observaram uma diminuição da atividade fagocítica de macrófagos alveolares em resposta a estímulo com $S$. cerevisie nos animais contidos por 40 minutos, tendo atribuído essa diminuição à presença de níveis elevados de corticosterona. De fato, o mecanismo subjacente pode estar relacionado com a presença aumentada desses hormônios e com a sua ação inibidora na expressão de receptores em macrófagos $^{12}$. A diminuição da atividade fagocítica de macrófagos tanto pode comprometer o mecanismo de defesa inato, como a sequência de eventos da resposta imune adaptativa à infecção ${ }^{25,26}$. A eficiência dos fagócitos parece ter uma profunda influência no aumento ou supressão da inflamação e, se há problemas na remoção e reparo dos tecidos no processo inflamatório, a autoimunidade ou o câncer podem advir ${ }^{25-27}$.

Os macrófagos são consumidores de glutamina, tanto para o seu metabolismo (síntese de purinas e de coenzimas) como para realizar suas funções ${ }^{27,28}$. Quando cultivados em meio com baixa concentração de glutamina, apresentam uma diminuição na capacidade de apresentar antígenos, produção de ânion superóxido, produção de citocinas e da atividade fagocítica de antígenos opsonizados ${ }^{25,27}$. No presente estudo, a administração in vivo de glutamina nos animais estressados não atenuou a diminuição da atividade fagocítica de macrófagos. É possível que outras células mais sensíveis aos efeitos do estresse possam aumentar a captação de glutamina da circulação ${ }^{10}$. Ou ainda que a glutamina suplementada nos dias anteriores possa ter sido utilizada por outros tecidos como, por exemplo, os adipócitos ${ }^{10}$. De fato, os animais deste estudo que receberam glutamina apresentaram um maior ganho de peso corporal, comparativamente aos seus pares que não a receberam. A velocidade de utilização da glutamina é influenciada por 
sua disponibilidade, por fatores hormonais, pelo transporte através da membrana e pela velocidade catalítica máxima da enzima glutaminase ${ }^{15}$. Taxas de utilização da glutamina, tão ou mais altas que a da glicose, ocorrem em muitas células ${ }^{15}$.

Este estudo fortalece as evidências dos efeitos imunossupressores do estresse motivado por fatores psicológicos em animais de laboratório. Esses efeitos foram verificados tanto relativamente à concentração de células imunes como à função fagocítica de macrófagos. Se essas alterações são transitórias, simplesmente reflexo de uma automodulação das células imunes em busca da homeostase, ou se são permanentes, comprometendo, dessa forma, a atuação dos componentes do sistema imune, é um assunto a ser considerado. Todavia, é bem provável que, mesmo de natureza transitória, essas alterações tenham consequências fisiopatológicas importantes e mereçam ser consideradas na etiologia de algumas doenças, como, por exemplo, o câncer.

Um dos fatores que pode influenciar a imunossupressão é a diminuição da disponibilidade de glutamina no plasma. Entretanto, este estudo verificou que, mesmo aumentando a disponibilidade de glutamina, não houve atenuação dos parâmetros imunes analisados.

\section{A GRADECIMENTOS}

Apoio financeiro da Fundação de Amparo à Ciência do Estado de Pernambuco (FACEPE) e do Conselho Nacional de Desenvolvimento Científico e Tecnológico (CNPq).

\section{REFERÊ NCIAS}

1. Ardawi MS, Newsholme EA. Metabolism in lymphocytes and its importance in the immune response. Essays Biochem. 1985; 21:1-44.

2. Calder PC, Yaqoob P. Glutamine and the immune system. Amino Acids. 1999; 17(3):227-41.

3. Fahr MJ, Kornbluth J, Blossom S, Schaeffer R, Limberg S. Glutamine enhances immunoregulation of tumor growth. JPEN. 1994; 18(6):471-6.
4. Wu G, Meininger CJ, Knabe DA, Bazer FW, Rhoads $\mathrm{JM}$. Arginine nutrition in development, health and disease. Curr Opin Clin Nutr Metab Care. 2000; 3(1):59-66.

5. Neu J, DeMarco V, Li N. Glutamine: clinical applications and mechanisms of action. Curr Opin Clin Nutr Metab Care. 2002; 5(1):69-75.

6. O'Flaherty L, Bouchier-hayes DJ. Immunomutrition and surgical practice. Proc Nutr Soc. 1999; 58(4)831-7.

7. Newsholme EA. Biochemical mechanisms to explain immunosuppression in well-trained and overtrained athletes. Int J Sports Med. 1994; 15(3 Suppl):S142-7.

8. Castell LM, Newsholme EA. Glutamine and the effects of exhaustive exercise upon the immune response. Can J Physiol Pharmacol. 1998; 76(5):524-32

9. Koyama K, Kaya M, Tsujita J, Hori S. Effects of decreased plasma glutamine concentrations on peripheral lymphocyte proliferation in rats. Eur J Appl Physiol Occup Physiol. 1998; 77(1-2):25-31.

10. Kew S, Wells SM, Yaqoob P, Wallace FA, Miles EA, Calder PC. Dietary glutamine enhances murine T-Lymphocyte responsiveness. J Nutr. 1999; 129(8):1524-31.

11. Heath GW, Ford ES, Craven TE, Macera CA, Jackson $K L$, Pate RR. Exercise and the incidence of upper respiratory tract infections. Med Sci Sports Exerc. 1991; 23(2):152-7.

12. Moinard C, Chauveau B, Walrand S, Felgines C, Chassagne J, Caldefie F, et al. Phagocyte functions in stressed rats: comparison of modulation by glutamine, arginine and ornithine 2-oxoglutarate. Clin Sci (Lond). 1999; 97(1):59-65.

13. Pithon-Curi TC, De Melo MP, Curi R. Glucose and glutamine utilization by rat lymphocytes, monocytes and neutrophils in culture: a comparative study. Cell Biochem Funct. 2004; 22(5):321-6.

14. Curi R, Newsholme P, Pithon-Curi TC, Pires-de-Melo M, Garcia C, Homem-de-Bbittencourt PIJR, et al. Metabolic fate of glutamine in lymphocytes, macrophages and neutrophils. Braz J Med Biol Res. 1999; 32(1):15-21.

15. Koretz RL. Immunonutrition: fact, fantasy, and future. Curr Gastroenterol Rep. 2002; 4(4):332-7.

16. Selye $H$. Thymus and adrenals in the response of the organism to injuries and Intoxication. Br J Exp Path. 1936; 17:234-9

17. Stibbe W, Weise M, Seidel D. Automated platelet count in thrombocytopenic patients-a comparison of methods. J Clin Chem Clin Biochem. 1985; 23(7):399-404 
18. De Castro CB, Manhães-de-Castro R, Medeiros AF Queirós A, Ferreira WT, Lima Filho JL. Effect of stress on the production of $\mathrm{O} 2$ - in alveolar macrophages. J Neuroimmunol. 2000; 108(1):68-72.

19. Malagueño E, Albuquerque C, Castro CMMB, Gadelha M, Irmão JI, Santana JV. Effect of Biomphalaria straminea Plasma in the Phagocytosis of Biomphalaria glabrata Hemolymph Cells. Mem Inst Oswaldo Cruz. 1998; 93(1):301-2

20. Gaillard RC. Neuroendocrine-Immune system interactions. The Immune-hypotalamo-pituitaryadrenal axis. Trends Endoc Metabol. 1994; 7(5):303-9.

21. Stefanski V, Engler H. Effects of acute and chronic social stress on blood cellular immunity in rats. Physiol Behav. 1998; 64(5):733-41.

22. Bauer ME, Perks P, Lightman SL, Shanks N. Restraint stress is associated with changes in glucocorticoid immunregulation. Physiol Behav. 2001; 73(4): 525-32.

23. Nascimento E, Cavalcante T, Pereira S, Palmeira A, Rocha MC, Viana MT, et al. O exercício físico crónico altera o perfil leucocitário e a taxa de fagocitose de ratos estressados. Rev Port Ciênc Desp. 2004; 4(3):26-33.

24. Nascimento E, Manhães-de-Castro R, Castro CB, Leandro CG. Pode a glutamina modular a imunidade? Anais Faculd Med CCS - UFPE. 2001; 46(2):67-70.

25. Zwilling BS, Hilburger ME. Macrophage resistance genes: Bcg/lty/Lsh Immunol Ser. 1994; 60:233-45.

26. Castell L. Glutamine supplementation in vitro and in vivo, in exercise and in immunodepression. Sports Med. 2003; 33(5):323-45.

27. Woods JA, Lu Q, Ceddia MA, Lowder T. Special feature for the Olympics: effects of exercise on the immune system: exercise-induced modulation of macrophage function. Int J Sports Med. 2000; 21(Suppl 1):24-30.

28. Leandro C, Nascimento E, Manhães-de-Castro R, Duarte J, de-Castro C. Exercício físico e sistema imunológico: mecanismos e integrações. Rev Port Ciênc Desp. 2002; 2(5):80-90.

Recebido em: 24/6/2005

Versão final reapresentado em: 18/1/2006 Aprovado em: 29/3/2006 